\title{
An XML-based document suite
}

\author{
Dietmar Rösner and Manuela Kunze \\ Otto-von-Guericke-Universität Magdeburg \\ Institut für Wissens- und Sprachverarbeitung \\ P.O.box 4120, 39016 Magdeburg, Germany \\ (roesner,makunze)@iws.cs.uni-magdeburg.de
}

\begin{abstract}
We report about the current state of development of a document suite and its applications. This collection of tools for the flexible and robust processing of documents in German is based on the use of XML as unifying formalism for encoding input and output data as well as process information. It is organized in modules with limited responsibilities that can easily be combined into pipelines to solve complex tasks. Strong emphasis is laid on a number of techniques to deal with lexical and conceptual gaps that are typical when starting a new application.
\end{abstract}

\section{Introduction}

We have designed and implemented the XDOC document suite as a workbench for the flexible processing of electronically available documents in German. We have decided to exploit XML (Bray et al., 1998) and its accompanying formalisms (e.g. XSLT (Site, 2002b)) and tools (e.g. xt (Clark, 2002) ) as a unifying framework. All modules in the XDOC system expect XML documents as input and deliver their results in XML format.

$\mathrm{XML}$ - and ist precursor SGML - offers a formalism to annotate pieces of (natural language) texts. To be more precise: If a text is (as a simple first approximation) seen as a sequence of characters (alphabetic and whitespace characters) then XML allows to associate arbitrary markup with arbitrary subsequences of contiguous characters. Many linguistic units of interest are represented by strings of contiguous characters (e.g. words, phrases, clauses etc.). To use XML to encode information about such a substring of a text interpreted as a meaningful linguistic unit and to associate this information directly with the occurrence of the unit in the text is a straightforward idea. The basic idea is further backed by XMLs demand that XML elements have to be properly nested. This is fully concordant with standard linguistic practice: complex structures are made up from simpler structures covering substrings of the full string in a nested way.

The end users of our applications are domain experts (e.g. medical doctors, engineers, ...). They are interested in getting their problems solved but they are typically neither interested nor trained in computational linguistics. Therefore the barrier to overcome before they can use a computational linguistics or text technology system should be as low as possible.

This experience has consequences for the design of the document suite. The work in the XDOC project is guided by the following design principles that have been abstracted from a number of experiments and applications with "realistic" documents (i.a. emails, abstracts of scientific papers, technical documentation, ...):

- The tools shall be usable for 'realistic' documents. One aspect of 'realistic' documents is that they typically contain domain-specific tokens that are not directly covered by classical lexical categories (like noun, verb, ...). Those tokens are nevertheless often essential for the user of the document (e.g. an enzyme descriptor like EC 4.1.1.17 for a biochemist).

- The tools shall be as robust as possible.

In general it can not be expected that lexicon information is available for all tokens in a document. This is not only the case for most tokens from 'nonlexical' types - like telephone numbers, enzyme names, material codes, ...--, even for lexical types there will always be 'lexical gaps'. This may either be caused by neologisms or simply by starting to process documents from a new application domain with a new sublanguage. In the latter case lexical items will typically be missing in the lexicon ('lexical gap') and phrasal structures may not or not adequately be covered by the grammar.

- The tools shall be usable independently but shall allow for flexible combination and interoperability.

- The tools shall not only be usable by developers but as well by domain experts without linguistic training.

Here again XML and XSLT play a major role: XSL stylesheets can be exploited to allow different presentations of internal data and results for different target groups; for end users the internals are in many cases not helpful, whereas developers will need them for debugging. 
The tools in the XDOC document suite can be grouped according to their function:

- preprocessing

- structure detection

- POS tagging

- syntactic parsing

- semantic analysis

- tools for the specific application: e.g. information extraction

In all tools the results of processing is encoded with XML tags delimiting the respective piece of text. The information conveyed by the tag name is enriched with XML attributes and their resp. values.

\section{Preprocessing}

Tools for preprocessing are used to convert documents from a number of formats into the XML format amenable for further processing. As a subtask this includes treatment of special characters (e.g. for umlauts, apostrophes, ...).

\section{Structure detection}

We accept raw ASCII texts without any markup as input. In such cases structure detection tries to uncover linguistic units (e.g. sentences, titles, ...) as candidates for further analysis. A major subtask is to identify the role of interpunction characters.

If we have the structures in a text explicitly available this may be exploited by subsequent linguistic processing. An example: For a unit classified as title or subtitle you will accept an NP whereas within a paragraph you will expect full sentences.

In realistic texts even the detection of possible sentence boundaries needs some care. A period character may not only be used as a full stop but may as well be part of an abbreviation (e.g. 'z.B.' - engl.: 'e.g.' - or 'Dr.'), be contained in a number (3.14), be used in an email address or in domain specific tokens. The resources employed are special lexica (e.g. for abbreviations) and finite automata for the reliable detection of token from specialized non-lexical categories (e.g. enzyme names, material codes, ...).

These resources are used here primarily to identify those full stop characters that function as sentence delimiters (tagged as IP). In addition, the information about the function of strings that include a period is tagged in the result (e.g. ABBR).

\section{Example 1 results of structure detection}

Anwesend $<$ IP $>:</$ IP $>$

$<$ ABBR $>$ Univ. - Prof. $</ A B B R>$

$<\mathrm{ABBR}>\mathrm{Dr} .</ \mathrm{ABBR}><\mathrm{ABBR}>$ med. $</ \mathrm{ABBR}>$ Dieter Krause $<$ IP $>,</$ IP $>$

Direktor des Institutes fuer Rechtsmedizin

\section{POS tagging}

To try to assign part-of-speech information to a token is not only a preparatory step for parsing. The information gained about a document by POS tagging and evaluating its results is valuable in its own right. The ratio of token not classifiable by the POS tagger to token classified may e.g. serve as an indication of the degree of lexical coverage.

In principle a number of approaches is usable for POS tagging (e.g. (Brill, 1992)). We decided to avoid approaches based on (supervised) learning from tagged corpora, since the cost for creating the necessary training data are likely to be prohibitive for our users (especially in specialized sublanguages).

The approach chosen was to try to make best use of available resources for German and to enhance them with additional functionality. The tool chosen is not only used in POS tagging but serves as a general morpho-syntactic component for German: MORPHIX.

The resources employed in XDOC's POS tagger are:

- the lexicon and the inflectional analysis from the morphosyntactic component MORPHIX

- a number of heuristics (e.g. for the classification of token not covered in the lexicon)

For German the morphology component MORPHIX (Finkler and Neumann, 1988) has been developed in a number of projects and is available in different realisations. This component has the advantage that the closed class lexical items of German as well as all irregular verbs are covered. The coverage of open class lexical items is dependent on the amount of lexical coding. The paradigms for e.g. verb conjugation and noun declination are fully covered but to be able to analyze and generate word forms their roots need to be included in the MORPHIX lexicon.

We exploit MORPHIX - in addition to its role in syntactic parsing - for POS tagging as well. If a token in a German text can be morphologically analysed with MORPHIX the resulting word class categorisation is used as POS information. Note that this classification need not be unique. Since the tokens are analysed in isolation multiple analyses are often the case. Some examples: the token 'der' may either be a determiner (with a number of different combinations for the features case, number and gender) or a relative pronoun, the token 'liebe' may be either a verb or an adjective (again with different feature combinations not relevant for POS tagging).

In addition since we do not expect extensive lexicon coding at the beginning of an XDOC application some tokens will not get a MORPHIX analysis. We then employ two techniques: We first try to make use of heuristics that are based on aspects of the tokens that can easily be detected with simple string analysis (e.g. upper/lowercase, endings, ...) and/or exploitation of the token position relative to sentence boundaries (detected in the structure detection module). If a heuristic yields a classi- 
fication the resulting POS class is added together with the name of the employed heuristic (marked as feature SRC, cf. example 3 ). If no heuristics are applicable we classify the token as member of the class unknown (tagged with $\mathrm{XXX).}$

To keep the POS tagger fast and simple the disambiguation between multiple POS classes for a token and the derivation of a possible POS class from context for an unknown token are postponed to syntactic processing. This is in line with our general principle to accept results with overgeneration when a module is applied in isolation (here: POS tagging) and to rely on filtering ambiguous results in a later stage of processing (here: exploiting the syntactic context).

Example 2 domain-specific tagging

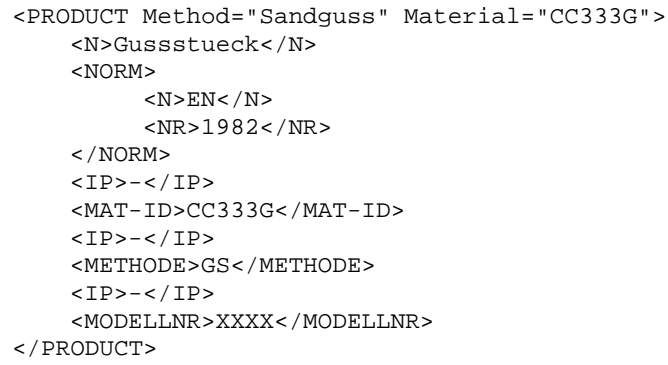

The example above is the result of tagging a domainspecific identifier. The token is annotated as a PROD$U C T$ with description of the used method and material. It is a typical token in the domain of casting technology.

\section{Syntactic parsing}

For syntactic parsing we apply a chart parser based on context free grammar rules augmented with feature structures.

Again robustness is achieved by allowing as input elements:

- multiple POS classes,

- unknown classes of open world tokens and

- tokens with POS class, but without or only partial feature information.

Example 3 unknown token classified as noun with heuristics

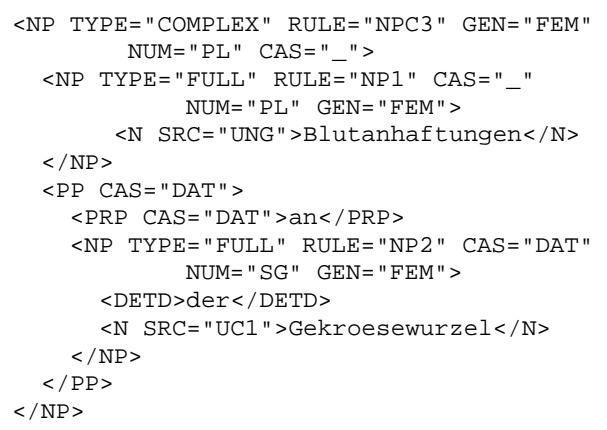

The latter case results from some heuristics in POS tagging that allow to assume e.g. the class noun for a token but do not suffice to detect its full paradigm from the token (note that there are ca two dozen different morphosyntactic paradigms for noun declination in German).

For a given input the parser attempts to find all complete analyses that cover the input. If no such complete analysis is achievable it is attempted to combine maximal partial results into structures covering the whole input.

A successful analysis may be based on an assumption about the word class of an initially unclassified token (tagged XXX). This is indicated in the parsing result (feature AS) and can be exploited for learning such classifications from contextual constraints. In a similar way the successful combination from known feature values from closed class items (e.g. determiners, prepositions) with underspecified features in agreement constraints allows the determination of paradigm information from successfully processed occurrences. In example 4 features of the unknown word "Mundhoehle" could be derived from the features of the determiner within the PP.

Example 4 unknown token classified as adjective and features derived through contextual constraints

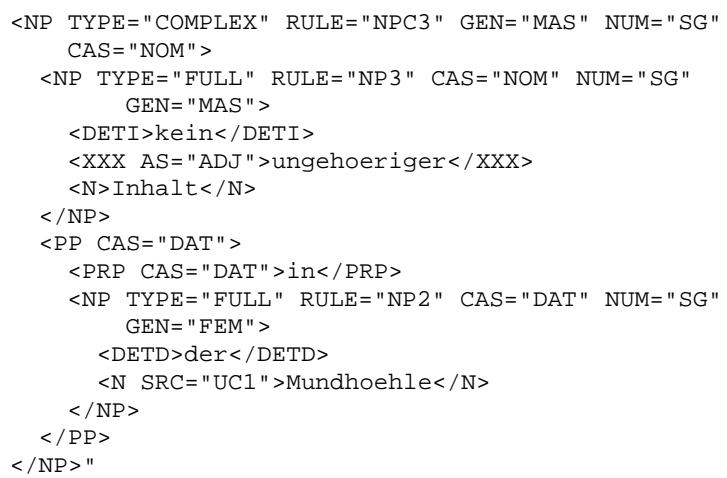

The grammar used in syntactic parsing is organized in a modular way that allows to add or remove groups of rules. This is exploited when the sublanguage of a domain contains linguistic structures that are unusual or even ungrammatical in standard German.

Example 5 Excerpt from syntactic analysis

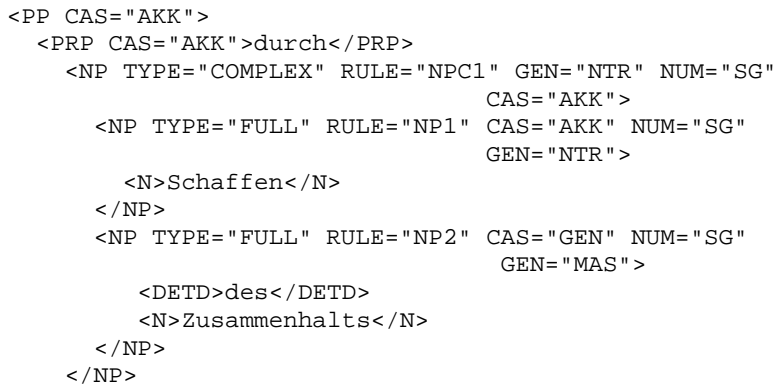




\section{Semantic analysis}

At the time of writing semantic analysis uses three methods:

\section{Semantic tagging}

For semantic tagging we apply a semantic lexicon. This lexicon contains the semantic interpretation of a token and a case frame combined with the syntactic valence requirements. Similar to POS tagging the tokens are annotated with their meaning and a classification in semantic categories like e.g. concepts and relations. Again it is possible, that the classification of a token in isolation is not unique. Multiple classification can be resolved through the following analysis of the case frame and through its combination with the syntactic structure which includes the token.

\section{Analysis of case frames}

By the case frame analysis of a token we obtain details about the type of recognized concepts (resolving multiple interpretations) and possible relations to other concepts. The results are tagged with XML tags. The following example describes the DTD for the annotation of the results of case frame analysis.

Example 6 DTD for the annotation by case frame analysis

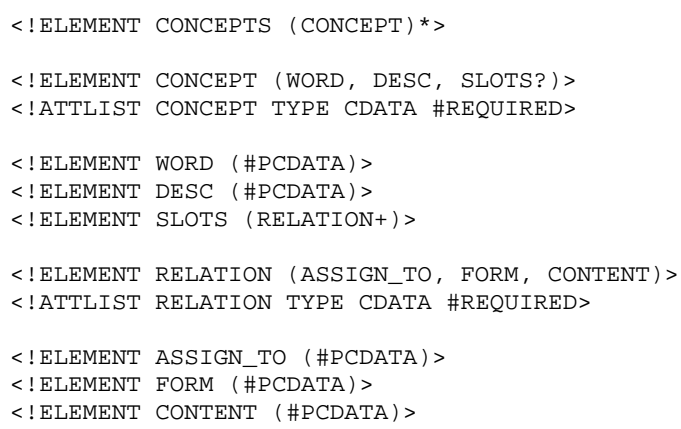

We use attributes to show the description of the concepts and we can annotate the relevant relations between the concepts through nested tags (e.g. the tag SLOTS).

Example 7 Excerpt from case frame analysis

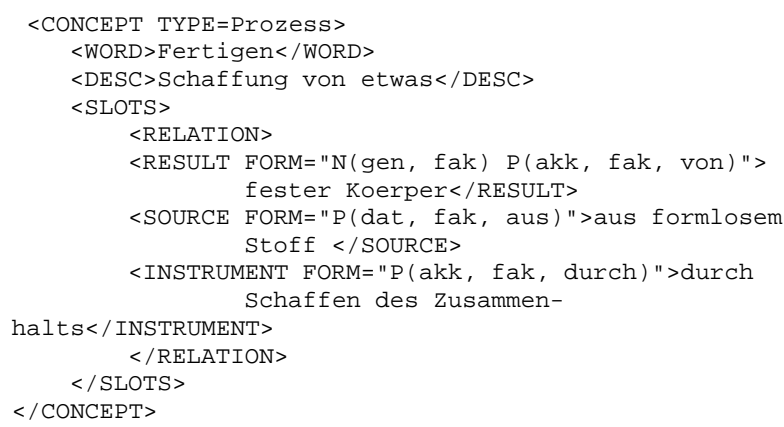

The example above is part of the result of the analysis of the German phrase: Fertigen fester Koerper aus formlosem Stoff durch Schaffen des Zusammenhalts ${ }^{1}$. The token Fertigen is classified as process with the relations source, result and instrument. The following phrases (noun phrases and preposition phrases) are checked to make sure that they are assignable to the relation requirements (semantic and syntactic) of the token Fertigen.

\section{Semantic interpretation of the syntactic structure}

An other step to analyze the relations between tokens can be the interpretation of the syntactic structure of a phrase or sentences respectively. We exploit the syntactic structure of the sublanguage to extract the relation between several tokens. For example a typical phrase from an autopsy report: Leber dunkelrot. ${ }^{2}$

From semantic tagging we obtain the following information:

\section{Example 8 results of semantic tagging}

$<$ CONCEPT TYPE="organ" $>$ Leber $</$ CONCEPT $>$ $<$ PROPERTY TYPE=" COlor" $>$ dunkelrot $</$ PROPERTY $>$ $\langle\mathrm{XXX}\rangle .\langle/ \mathrm{XXX}\rangle$

In this example we can extract the relation "has-color" between the tokens Leber and dunkelrot. This is an example of a simple semantic relation. Other semantic relations can be described through more complex variations. In these cases we must consider linguistic structures like modifiers (e.g. etwas), negations (e.g. nicht), coordinations (e.g. Beckengeruest unversehrt und fest gefuegt) and noun groups (e.g. Bauchteil der grossen Koerperschlagader).

\section{Current state and future work}

The XDOC document workbench is currently employed in a number of applications. These include:

- knowledge acquisition from technical documentation about casting technology

- extraction of company profiles from WWW pages

- analysis of autopsy protocols

The latter application is part of a joint project with the institute for forensic medicine of our university. The medical doctors there are interested in tools that help them to exploit their huge collection of several thousand autopsy protocols for their research interests. The confrontation with this corpus has stimulated experiments with 'bootstrapping techniques' for lexicon and ontology creation.

The core idea is the following:

When you are confronted with a new corpus from a new domain, try to find linguistic structures in the text that are easy to detect automatically and that allow to

\footnotetext{
${ }^{1}$ In English: production of solid objects from formless matter by creating cohesion

${ }^{2}$ In English: Liver dark red.
} 
classify unknown terms in a robust manner both syntactically as well as on the knowledge level. Take the results from a run of these simple but robust heuristics as an initial version of a domain dependent lexicon and ontology. Exploit these initial resources to extend the processing to more complicated linguistic structures in order to detect and classify more terms of interest automatically.

An example: In the sublanguage of autopsy protocols (in German) a very telegrammatic style is dominant. Condensed and compact structures like the following are very frequent:

\section{Harnblase leer. \\ Harnleiter frei. \\ Nierenoberflaeche glatt. \\ Vorsteherdruese altersentsprechend. \\ ...}

These structures can be abstracted syntactically as $<$ Noun $><$ Adjective $><$ Fullstop $>$ and semantically as reporting a finding in the form <Anatomic-entity $>$ has <Attribute-value $>$ and they are easily detectable (Rösner and Kunze, 2002).

In our experiments we have exploited this characteristic of the corpus extensively to automatically deduce an initial lexicon (with nouns and adjectives) and ontology (with concepts for anatomic regions or organs and their respective features and values). The feature values were further exploited to cluster the concept candidates into groups according to their feature values. In this way container like entities with feature values like 'leer' (empty) or 'gefuellt' (full) can be distinguished from e.g. entities of surface type with feature values like 'glatt' (smooth).

\section{Related Work}

The work in XDOC has been inspired by a number of precursory projects:

In GATE (Site, 2002a; Cunningham and Wilks, 1988) the idea of piping simple modules in order to achieve complex functionality has been applied to NLP with such a rigid architecture for the first time. The project LT XML has been pioneering XML as a data format for linguistic processing.

Both GATE and LT XML ((LTG), 1999) were employed for processing English texts. SMES (Neumann et al., 1997) has been an attempt to develop a toolbox for message extraction from German texts. A disadvantage of SMES that is avoided in XDOC is the lack of a uniform encoding formalism, in other words, users are confronted with different encodings and formats in each module.

\section{System availability}

Major components of XDOC are made publicly accessible for testing and experiments under the URL:

http://lima.cs.uni-magdeburg.de:8000/

\section{Summary}

We have reported about the current state of the XDOC document suite. This collection of tools for the flexible and robust processing of documents in German is based on the use of XML as unifying formalism for encoding input and output data as well as process information. It is organized in modules with limited responsibilities that can easily be combined into pipelines to solve complex tasks. Strong emphasis is laid on a number of techniques to deal with lexical and conceptual gaps and to guarantee robust systems behaviour without the need for a priori investment in resource creation by users. When end users are first confronted with the system they typically are interested in quick progress in their application but should not be forced to be engaged e.g. in lexicon build up and grammar debugging, before being able to start with experiments. This is not to say that creation of specialized lexicons is unnecessary. There is a strong correlation between prior investment in resources and improved performance and higher quality of results. Our experience shows that initial results in experiments are a good motivation for subsequent efforts of users and investment in extended and improved linguistic resources but that a priori costs may be blocking the willingness of users to get really involved.

\section{References}

Tim Bray, Jean Paoli, and C.M. Sperberg-McQueen. 1998. Extensible Markup Language (XML) 1.0. http://www.w3.org/TR/1998/REC-xml-19980210.

E. Brill. 1992. A simple rule-based part-of-speech tagger. In Proceeding of the Third Conference on Applied Natural Language Processing, pages 152-155.

J. Clark. 2002. http://www.jclark.com.

H. Cunningham and Y. Wilks. 1988. GATE - a General Architecture for Text Engineering. Proccedings of COLING-96. http://gate.ac.uk.

W. Finkler and G. Neumann. 1988. MORPHIX: a fast Realization of a classification-based Approach to Morphology. In H. Trost, editor, Proc. der 4. Österreichischen Artificial-Intelligence Tagung, Wiener Workshop Wissensbasierte Sprachverarbeitung, pages 11-19. Springer Verlag, August.

Language Technology Group (LTG). 1999. LT XML version 1.1. http://www.ltg.ed.ac.uk/software/xml/.

G. Neumann, R. Backofen, J. Baur, M. Becker, and C. Braun. 1997. An information extraction core system for real world german text processing. pages 208215, March.

D. Rösner and M. Kunze. 2002. Exploiting sublanguage and domain characteristics in a bootstrapping approach to lexicon and ontology creation. In Proceedings of the OntoLex 2002 - Ontologies and Lexical Knowledge Bases at the LREC 2002.

GATE Site. 2002a. http://gate.ac.uk.

XSL Site. 2002b. http://www.w3.org/style/xsl. 